\title{
Analysis of the Improvement of the Carbon Fiber Mono-filaments Tensile Strength in Tensile Device
}

\author{
Jiabin $\mathrm{Xu}^{1}$, Guixin $\mathrm{Zhao}^{2}$, Haitao $\mathrm{Wen}^{3}$, Zhaoqing Dong, Daigen Chen \\ ${ }^{1}$ Jiabin Science Laboratory, Xi'an, 710308, China \\ ${ }^{2}$ Project Cost Management Center, Second Oil Production Plant, Petrochina Daqing Oilfield, Daqing, 163414, China \\ ${ }^{3}$ School of Automotive Engineering, Harbin Institute of Technology, Weihai, 264209, China
}

\begin{abstract}
This paper describes a new micro-scale machining process which experimentally demonstrated a carbon fiber mono-filament tensile nanofibers and micro-scale devices fixture based on a $180^{\circ}$ - and $90^{\circ}$ - orthogonality carbon fiber mono-filament tensile (OCFMT). The fabricated OCFMTs with orthogonality structures at $180^{\circ}$ and $90^{\circ}$ consecutively in carbon fiber mono-filament. When the vertical load applied to the $90^{\circ}$ - OCFMT, the micro-scale devices fixture will effective eliminate of the gravity tilt angles with an offset value effects. The fabricated OCFMTs interchange from each other angle, which provides a solution to measure the fixture. The experimental consequence clearly show that obtain effective tensile mechanical property can be developed based on the proposed OCFMT nano- and micro-scale devices and systems in application fields such as tensile strength testing of carbon fiber mono-filament , nanofibers, carbon nanofibers, bio-medical, high polymer.
\end{abstract}

Keywords- carbon fiber mono-filament tensile, canofibers, nano- and micro-scale devices, fixture.

\section{INTRODUCTION}

In the present work, carbon fiber monofilaments are one of the high performance fibers applied in the advanced composites. Tensile strength of carbon fibers based carbon fiber monofilaments were tested for $180^{\circ}$ and $90^{\circ}$ - orthogonality gauge lengths. External tiny shake also influence the carbon fiber mono-filament tensile accuracy. In this paper, it was found that the fixture size effect in horizontal and vertical direction was almost similar for all fibers tested.

Additional split-collet tensile grips and holders system is analyzed from the carbon fibers tensile strength point of view. The carbon fiber monofilaments are although all thin, still it have a wide variety in thicknesses and tensile strength. The carbon fiber mono-filament tensile nanofibers and micro-scale devices fixture based on a $180^{\circ}$ - and $90^{\circ}$ - orthogonality carbon fiber mono-filament tensile (OCFMT) need very particular gripping methods. The horizontal and vertical direction grips provide the ideal means to hold single fiber specimens. These grips have an adjustable clamping force, self-aligning tilt angles and provide effective of operation similar to the carbon fiber mono-filaments.

At the composites strength split-collet tensile grips and holders design, the carbon fiber monofilaments strengths evaluated from the fibers strands with a certain gauge length are usually used. Tensile strength of carbon fibers, however, shows a large scatter and remarkable size dependence according to the weakest link analogy.

For instance, the carbon fiber mono-filament tensile (OCFMT) in tensile device will be more effective improvement tensile strength of carbon fibers. Also, the tensile strength evaluated for a certitude gauge length in a generality as a measure of fiber strength.

\section{ORgANIZATION Of THE TEXT}

Most carbon fibers tensile strength proposed so far are based on effect operation with additional split-collet tensile grips and holders. The split-collet types shown in Figure 1 and Figure 2. This particular set of grips is designed for testing carbon fibers.

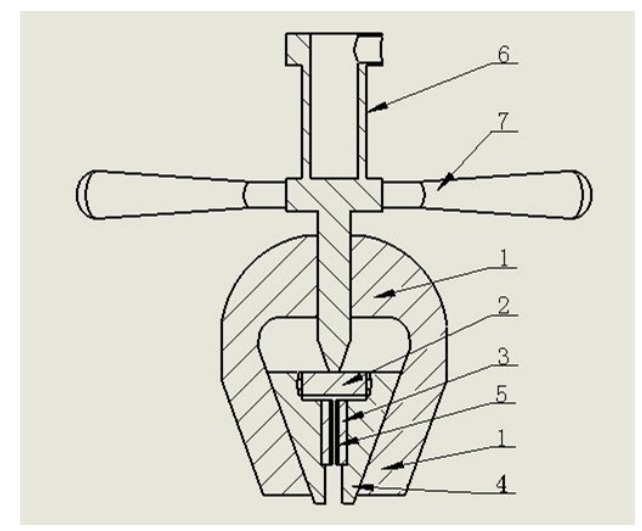

Figure 1: Design chart of the tensile device

Further illustration of the carbon fiber mono-filament tensile combined with figures: 1-body of tensile device; 2-tensile holder; 3-gauge block; 4-spilt-collet tensile grips; 5-carbon fiber; 6-ejector pin; 7-swivel arm (refer with: Fig. 2).

The carbon fiber mono-filament tensile (OCFMT) strength testing of the strands of different tensile strength revealed compatible behavior with that of the carbon fiber mono-filament tensile nanofibers just outlined. OCFMT and micro-scale devices fixture process is cost effective and high stability. For a prototype fixture $(90 \mathrm{~mm}$ wide, 
$100 \mathrm{~mm}$ long, $20 \mathrm{~mm}$ thick) the measured maximum tip displacement was approximately $25 \mathrm{~mm}$.

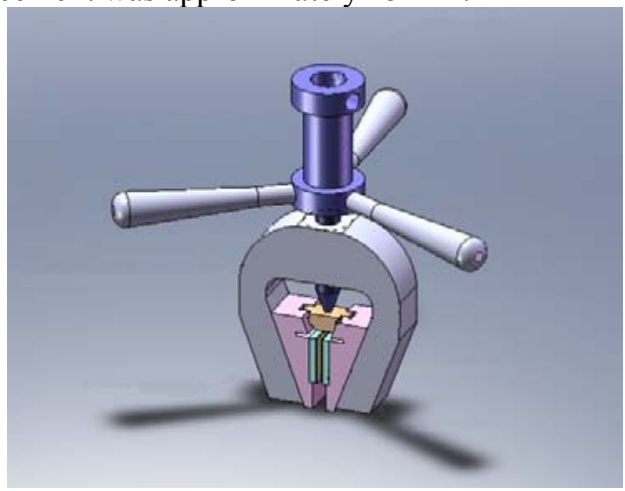

Figure 2: The perspective view of the tensile device

The fiber test grips design is then improved further in carbon fiber mono-filaments tensile. Based on the spilt-collet tensile grips and holders, the carbon fiber mono-filaments could effective tensile. When the vertical load applied to the $90^{\circ}$ - OCFMT, the micro-scale devices fixture will effective eliminate of the gravity title angle with an offset value effects.

\section{THEORETICAL CALCULATIONS}

\begin{tabular}{|c|c|c|c|}
\hline \multicolumn{4}{|c|}{ Symbols and their meanings } \\
\hline$P$ & Bolt pressure & $N$ & Supporting force \\
\hline$\alpha$ & Dip angle & $W$ & Pre-tightening force \\
\hline$\overline{f_{N}}$ & Friction force & $\bar{f}$ & Friction coefficient \\
\hline
\end{tabular}

TABLE 1: THEORETICAL CALCULATIONS' SYMBOLS AND THEIR MEANINGS

Step1: According to energy conservation, the stress analysis of the equilibrium state of spilt-collet tensile grips can be calculated (refer with: Eq. 1).

$$
\begin{aligned}
& W+f_{N} \sin \alpha=N \cos \alpha \\
& \frac{P}{2}=f_{N} \cos \alpha+N \sin \alpha
\end{aligned}
$$

Step2: The supporting force $\mathrm{N}$ can be calculated (refer with: Eq. 2).

$$
N=\frac{P}{2(f \cos \alpha+\sin \alpha)}
$$

Step3: Put Eq. 2 into Eq. 1, the pre-tightening force can be calculated (refer with: Eq. 3).

$$
W=\frac{P}{2} \cdot \frac{\cos \alpha-f \sin \alpha}{f \cos \alpha+\sin \alpha}=\frac{P}{2} \cdot \frac{1-f \tan \alpha}{f+\tan \alpha}
$$

So with $\alpha$ increase, $\mathrm{W}$ decrease. If we make $\alpha=0, \mathrm{~W}$ is the largest. The design of the spilt-collet tensile grips can be calculated reasonable.

\section{CONCLUSIONS}

The overall conclusion is that the carbon fiber mono-filament tensile (OCFMT) exhibited the characteristic cost effective and high stability but high adaptability nature of OCFMTs at room temperature. The micro-scale devices fixture properties were based on carbon fiber mono-filament diameter. The spilt-collet tensile grips and holders were very accurately predicts the carbon fiber mono-filaments length (or size) dependence of tensile strength. The clean, hard the carbon fiber mono-filaments tensile strength of OCFMTs presented the best effective and high stability.

\section{REFERENCES}

[1] W. Liu, Y. Shen and C. Lu, Differential Thread Principle's Application in Manual Drawing Device, Applied Mechanics and Materials Vol. 385-386, pp. 329, 2013.

[2] Y.Z Shen, Design of the Two-way Hydraulic Puller, Agricultural Mechanization Research Vol. 31, pp. 10, 2009.

[3] E.P.S. Tan, S.Y. Ng and C.T. Lim: Tensile Testing of A Single Ultrafine Polymeric Fiber, Biomaterials Vol. 26, pp. 1453, 2005.

[4] X. Ding etc. : Machining with Micro-size Single Crystalline Diamond Tools Fabricated by A Focused Ion Beam, Micromechanics and Microengineering Vol. 19, pp. 1, 2009.

[5] Shun-Tong Chen: A High-efficiency Approach for Fabricating Mass Micro Holes by Batch Micro EDM, Micromechanics and Microengineering Vol. 17, pp. 1961, 2007.

[6] W. Liu etc. : Analysis of the Improvement of the Drawing Die in Drawing Device, Applied Mechanics and Materials Vol. 496-500, pp. 1041, 2014. 